POS PROCEEDINGS

\title{
JLQCD's dynamical overlap project
}

JLQCD Collaboration: T. Kaneko ${ }^{* a, b} ;$ S. Aoki ${ }^{c}$, H. Fukaya ${ }^{d}$, S. Hashimoto ${ }^{a, b}$, K-I. Ishikawa ${ }^{e}$, K. Kanaya ${ }^{c}$, H. Matsufuru ${ }^{a}$, M. Okamoto ${ }^{a}$, M. Okawa ${ }^{e}$, T. Onogi ${ }^{g}$, A. Ukawa ${ }^{f, c}$, N. Yamada ${ }^{a, b}$, T. Yoshié $f, c$

${ }^{a}$ High Energy Accelerator Research Organization (KEK), Ibaraki 305-0801, Japan

${ }^{b}$ School of High Energy Accelerator Science, The Graduate University for Advanced Studies

(Sokendai), Ibaraki 305-0801, Japan

${ }^{c}$ Graduate School of Pure and Applied Sciences, University of Tsukuba, Ibaraki 305-8571, Japan

${ }^{d}$ Theoretical Physics Laboratory, RIKEN, Saitama 351-0198, Japan

e Department of Physics, Hiroshima University, Hiroshima 739-8526, Japan

${ }^{f}$ Center for Computational Sciences, University of Tsukuba, Ibaraki 305-8577, Japan

${ }^{g}$ Yukawa Institute for Theoretical Physics, Kyoto University, Kyoto 606-8502, Japan

We present an overview of our project of large-scale simulations with dynamical overlap fermions. The first production run in two-flavor QCD is on-going using the Iwasaki gauge action on a $16^{3} \times 32$ lattice at the lattice spacing of $0.12 \mathrm{fm}$ with six sea quark masses down to $m_{s, \text { phys }} / 6$, where $m_{s \text {,phys }}$ is the physical strange quark mass. We briefly introduce our choice of the lattice action and simulation algorithm, and describe the present status of the production run. Preliminary results on the light meson masses and the static quark potential are also reported.

XXIVth International Symposium on Lattice Field Theory

July 23-28, 2006

Tucson, Arizona, USA

\footnotetext{
*Speaker.

†E-mail: takashi.kaneko@kek.jp
} 


\section{Introduction}

The JLQCD Collaboration has pursued detailed studies of non-perturbative properties of QCD through numerical simulations on the supercomputer system at KEK. Our previous unquenched simulations $[1,2]$ however were restricted to rather heavy sea quark masses $\gtrsim m_{s \text {,phys }} / 2$, which make the chiral extrapolation difficult to control. The use of Wilson-type quarks also limits the applicability of our simulation to quantities, such as $B_{K}$, for which the explicit chiral symmetry breaking causes complicated operator mixing.

This year, the supercomputer system at KEK was replaced by a multiple system of Hitachi SR11000 and IBM Blue Gene/L with a total peak speed of about 60 TFLOPS. On this new system, we carry out large-scale simulations including dynamical overlap fermions with sea quark masses down to $m_{s, \text { phys }} / 4$ or smaller on reasonably fine $(a \lesssim 0.125 \mathrm{fm})$ and large lattices $(L \gtrsim 2 \mathrm{fm})$. In this article, we present an overview of our first production simulation in two-flavor QCD.

\section{Simulation method}

Our numerical simulations are carried out using the overlap quark action with the standard Wilson kernel $H_{W}$

$$
D\left(m_{\text {sea }}\right)=\left(m_{0}+\frac{m_{\text {sea }}}{2}\right)+\left(m_{0}-\frac{m_{\text {sea }}}{2}\right) \gamma_{5} \operatorname{sgn}\left[H_{W}\left(-m_{0}\right)\right],
$$

where we set $m_{0}=1.6$. A major problem with this kernel is the appearance of (near-)zero modes of $H_{\mathrm{W}}$ in simulations on relatively coarse lattices. This possibly spoils the locality of $D$ and also makes simulations costly. From our preparatory study in quenched QCD [3], we adopt the Iwasaki gauge action, with which the near-zero modes are suppressed compared to the standard plaquette glue and the localization range of $D$ is sufficiently small.

The use of the improved gauge action does not completely rule out the appearance of exact zero modes. In order to avoid the time consuming reftection/refraction procedure [4], we introduce twofhvors of unphysical extra-Wilson fermions with a large negative mass $-m_{0}[5,6]$ and additional twisted mass ghosts [6] which produce the Boltzmann weight

$$
\frac{\operatorname{det}\left[H_{\mathrm{W}}\left(-m_{0}\right)^{2}\right]}{\operatorname{det}\left[H_{\mathrm{W}}\left(-m_{0}\right)^{2}+\mu^{2}\right]} .
$$

The factor in the numerator suppresses the zero modes during continuous updating of gauge fields, such as the hybrid Monte Carlo (HMC) algorithm. The denominator with appropriately chosen twisted mass $\mu$ cancels possibly large effects from high modes of $H_{W}$ in the numerator. We note that these unphysical extra-fields have masses of cutoff order, and hence their effects vanish in the continuum limit. The suppression of zero modes in quenched and two-fhvor QCD is demonstrated in Refs.[6, 7].

While this procedure fixes the net topological charge $Q$ during the HMC update, it does not forbid local topological fluctuations. It is expected that, in the infinite volume limit, properties of hadrons with their size of $O(1 \mathrm{fm})$ are insensitive to the global topological charge of gauge configurations, i.e. the effect due to the fixed topology is a finite size effect (FSE). We refer to 
Ref.[7] for more detailed discussions. We also note that our lattice action to fix the topology provides a convenient framework for studies in the $\varepsilon$-regime [8].

In our simulation program, we implement multiplications of $D$ employing the low mode preconditioning: namely, we introduce a threshold $\lambda_{\text {th }}$ in the spectrum of $H_{\mathrm{W}}$, and eigenvalues smaller than $\lambda_{\text {th }}$ are projected out in the evaluation of $\operatorname{sgn}\left[H_{W}\right]$. For higher modes, we use the rational approximation with the Zolotarev coefficients and the multi-shift CG algorithm for the inner-loop [9]. For multiplications of $D^{-1}$, we employ a nested solver with the relaxed CG [10] for the outer-loop.

In our implementation of HMC, we employ a combination of the Hasenbusch preconditioning [11] and the multiple time scale discretization of the molecular dynamics (MD) [12], which has been shown to be effective in simulations with Wilson-type fermions [13]. The determinant factor for overlap quarks is written as

$$
\operatorname{det}\left[D\left(m_{\text {sea }}\right)^{2}\right]=\operatorname{det}\left[D\left(m^{\prime}\right)^{2}\right] \operatorname{det}\left[\frac{D\left(m_{\text {sea }}\right)^{2}}{D\left(m^{\prime}\right)^{2}}\right]
$$

where $m^{\prime}$ is the mass of the Hasenbusch preconditioner. Two pseudo-fermions, which we call PF1 and PF2 in the following, are introduced for the first and second factors in the r.h.s. Then, PF2 is updated $N_{\mathrm{MD}}^{(\mathrm{PF} 2)}$ times per unit trajectory length. The updates of PF1 and gauge fields are more frequent by factors of $R_{\mathrm{MD}}^{(\mathrm{PF})}$ and $R_{\mathrm{MD}}^{(\mathrm{PF})} R_{\mathrm{MD}}^{(\mathrm{G})}$. Since we suppress zero modes by the extra-Wilson fermion, we switch off the reflection/refraction procedure in our MD evolution.

In addition to the above algorithmic techniques, we also use an assembler code developed by IBM for multiplications of $D_{W}$ on Blue Gene/L. This code makes the best use of the double FPU instructions, which process double precision complex number arithmetic effectively using two sets of fbating-point registers. This assembler code is a factor of 3 faster than our naive Fortran code. Further details of our simulation algorithm are presented in Ref. [14].

\section{Production run}

Our first production run with two-flavors of degenerate up and down quarks is being carried out with the above mentioned lattice action. The twisted mass $\mu=0.2$ is chosen from a preparatory study in quenched QCD [6]. We simulate $\beta=2.30$, which is expected to correspond to the lattice spacing about $0.125 \mathrm{fm}$, on a $16^{3} \times 32$ lattice. Six sea quark masses listed in Table 1 are taken to

\begin{tabular}{l|llll|lll|ll}
\hline$m_{\text {sea }}$ & $m^{\prime}$ & $N_{\mathrm{MD}}^{(\mathrm{PF})}$ & $R_{\mathrm{MD}}^{(\mathrm{PF})}$ & $R_{\mathrm{MD}}^{(\mathrm{G})}$ & HMC traj. & $P_{\mathrm{HMC}}$ & time[min $]$ & \#conf $_{\text {pot }}$ & \#conf $_{\text {had }}$ \\
\hline 0.015 & 0.2 & 9 & 4 & 5 & 2150 & 0.89 & 6.1 & 151 & 173 \\
0.025 & 0.2 & 8 & 4 & 5 & 4320 & 0.90 & 4.7 & 389 & 410 \\
0.035 & 0.4 & 6 & 5 & 6 & 4150 & 0.74 & 3.0 & 411 & 403 \\
0.050 & 0.4 & 6 & 5 & 6 & 3500 & 0.79 & 2.6 & 310 & 310 \\
0.070 & 0.4 & 5 & 5 & 6 & 3500 & 0.81 & 2.1 & 307 & 243 \\
0.100 & 0.4 & 5 & 5 & 6 & 3590 & 0.85 & 2.0 & 301 & 319 \\
\hline
\end{tabular}

Table 1: Simulation parameters for configuration generation. Statistics for the measurements of static potential $\left(\#\right.$ conf $\left.{ }_{\text {pot }}\right)$ and hadron correlators $\left(\#\right.$ conf $\left.f_{\text {had }}\right)$ are also listed. 
explore a range from $m_{s, \text { phys }}$ down to $m_{s, \text { phys }} / 6$. All results in this article are obtained in the trivial topological sector $Q=0$.

In all simulations, we fix $\lambda_{\text {th }}=0.045$, with which several eigenvectors are projected out in the calculation of $\operatorname{sgn}\left[H_{\mathrm{W}}\right]$. The number of poles in the Zolotarev approximation is set to 10 leading to an accuracy of $\left|\operatorname{sgn}\left[H_{\mathrm{W}}\right]^{2}-1\right| \simeq 10^{-(7-8)}$ throughout the simulations. The stopping condition of the overlap solver is chosen so that the reversibility in the gauge field and the Hamiltonian is satisfied to a level comparable to our previous simulations.

We are generating gauge configurations on Blue Gene/L and store them on disks every 10

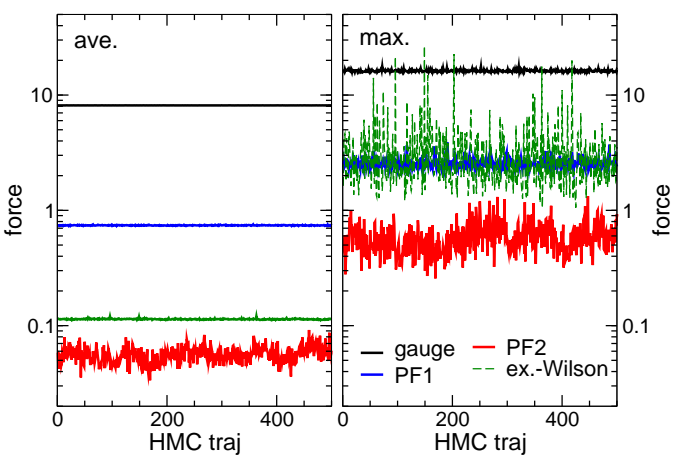

Figure 1: Average (left panel) and maximum value (right panel) of MD force at each trajectory. Data at $m_{\text {sea }}=0.015$ are plotted. trajectories. The HMC trajectory length is fixed to 0.5. Hadron correlators and the static quark potential are measured on both of SR11000 and Blue Gene/L using the stored configurations. Current statistics are summarized in Table 1.

Figure 1 shows an example of the time history of the MD force from gauge fields, PF1 and PF2. With our choice of $m^{\prime}$, these forces are well separated from each other. This situation suitable for the application of the multiple time scale discretization is confirmed also at other sea quark masses. In the same figure, we also plot the force from the extra-fields in Eq. (2.2). Its maximum value becomes as large as that for the gauge field probably due to small eigenvalues of $H_{\mathrm{W}}$. Therefore, we update the extra-fermions in the inner most MD step.

As summarized in Table 1, we achieve the acceptance rate $P_{\mathrm{HMC}}$ of $74 \%$ or higher with our choice of the MD discretization parameters. The CPU time per 1 trajectory on the whole machine of Blue Gene/L (10 racks, 57.3 TFLOPS) is also listed in the table. It takes about one month to accumulate 2000 trajectories at six sea quark masses. We have recently switched to a five dimensional solver proposed in [15], which reduces the CPU time by roughly a factor of 2 for our simulation parameters.

In Fig. 2, we plot the sea quark mass depen-

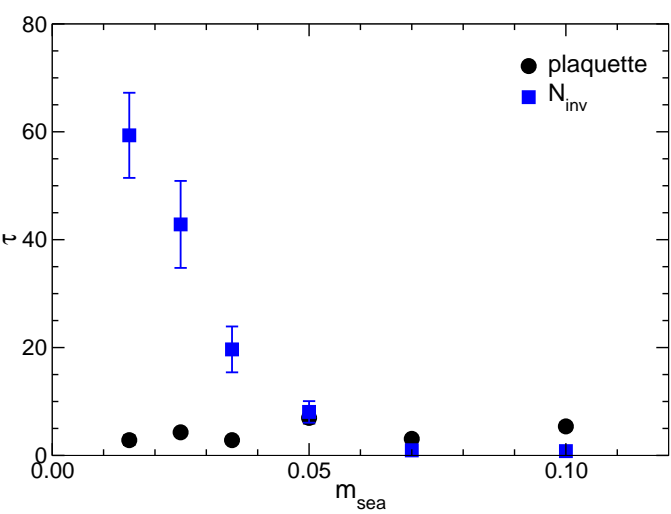

Figure 2: Autocorrelation time for plaquette and iteration count of the overlap solver $N_{\text {inv }}$. dence of the autocorrelation time for two quantities: $\tau_{\text {plaq }}$ for the plaquette, and $\tau_{\text {inv }}$ for the iteration count of the overlap solver $N_{\text {inv }}$. We find that $\tau_{\text {plaq }}$ is small and has a mild mass dependence, probably because it is a local quantity. On the other hand, $N_{\text {inv }}$ is expected to be sensitive to the low modes of $D$. In fact, $\tau_{\text {inv }}$ increases rapidly towards the chiral limit.

We find that $\tau_{\text {inv }} \approx 60$ at our lightest sea quark mass. This implies that statistics of $O(10,000)$ trajectories are needed for precise calculation of hadronic observables at such light quark masses. In the following, we present preliminary results with limited statistics listed in Table 1. 

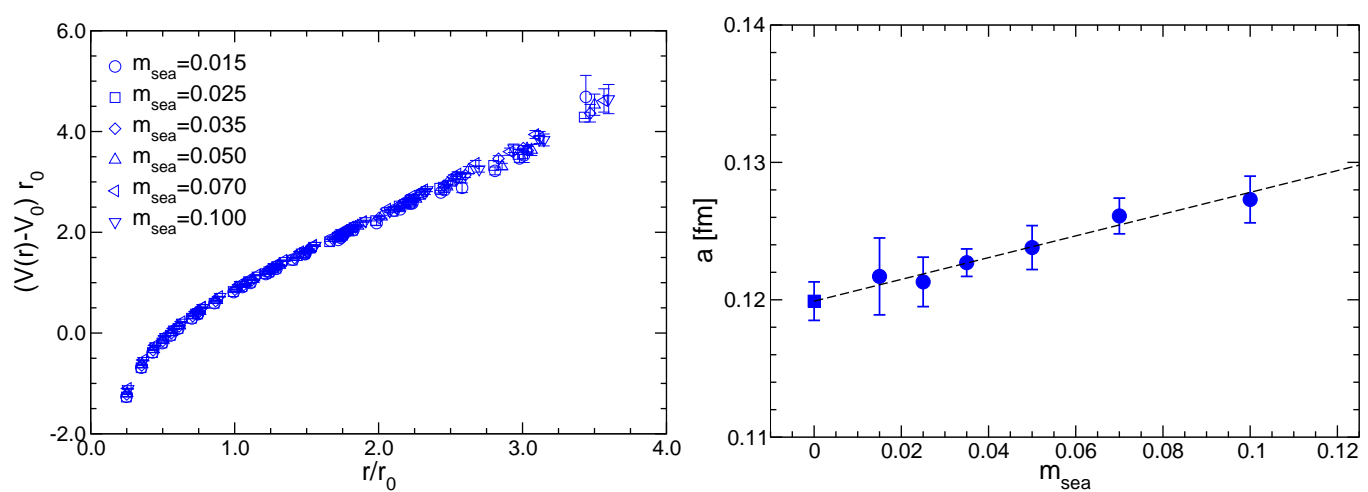

Figure 3: Plot of $\left(V(r)-V_{0}\right) r_{0}$ as a function of $r / r_{0}$ (left figure), and chiral extrapolation of lattice spacing determined from $r_{0}$ (right figure).

\section{Static quark potential}

We calculate the static quark potential $V(r)$ from the smeared Wilson loops. The Sommer scale $r_{0}[16]$ is extracted from a parametrization $V(r)=V_{0}-\alpha / r+\sigma r$.

In Fig. 3, we plot $V(r)$ rescaled by $r_{0}$ at all sea quark masses. While we simulate a wide range of the sea quark mass, all data form a universal curve. We note that our statistics are not sufficiently high to observe a clear dependence of $\alpha$ on the sea quark mass. The chiral extrapolation of the lattice spacing determined from $r_{0}$ is shown in the same figure. From a simple linear fit and an input $r_{0}=0.49 \mathrm{fm}$, we obtain $a=0.1199(14) \mathrm{fm}$ in the chiral limit, which is close to our target value $0.125 \mathrm{fm}$.

\section{Meson masses and decay constant}

Meson masses are extracted from correlation functions measured with smeared source and local sink operators. Figure 4 shows the chiral extrapolation for the pseudo-scalar and vector mesons made of degenerate valence quarks. With our statistics, we do not observe any significant deviation from simple linear fits

$$
M_{\mathrm{PS}}^{2}=b_{\mathrm{PS}} m_{\mathrm{sea}}, \quad M_{\mathrm{V}}=a_{\mathrm{V}}+b_{\mathrm{V}} M_{\mathrm{PS}}^{2},
$$
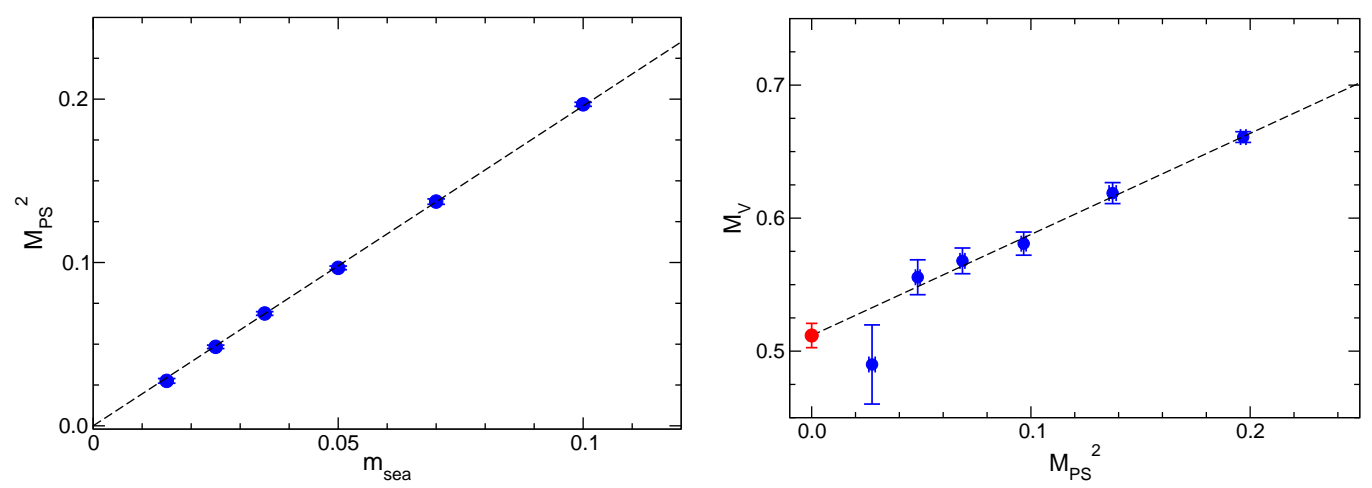

Figure 4: Chiral extrapolation of pseudo-scalar (left figure) and vector meson masses (right figure). 
which give $\chi^{2} /$ dof $\lesssim 1.0$. Employing this linear fit, we obtain $a=0.1312(23)$ fm from the $\rho$ meson mass input. This is consistent with the estimate from $r_{0}$ within $10 \%$ accuracy.

Since we are exploring small sea quark mass regime with a fixed topology, we have to be careful about FSEs. These are however difficult to assess reliably without performing direct simulations with different lattice volumes. Here we only note that an analytic formula for FSE [17] combined with an analysis for the fixed topology [18] suggests that FSEs are not large $(\approx 1-2 \%)$ even at our lightest sea quark mass.

In order to check the size of the chiral symmetry breaking, we calculate the following ratio motivated from the axial ward identity

$$
\rho_{\text {AWI }}=\frac{\left\langle\nabla_{4} A_{4} P^{\dagger}\right\rangle}{\left\langle P P^{\dagger}\right\rangle},
$$

where $P$ and $A_{\mu}$ are the pseudo-scalar and axial current operators. Fig. 5 shows the chiral extrapolation of $\rho_{\mathrm{AWI}}$ with the following linear and quadratic forms:

$$
\rho_{\mathrm{AWI}}=a_{\mathrm{AWI}}+b_{\mathrm{AWI}} m_{\mathrm{sea}}\left(+c_{\mathrm{AWI}} m_{\mathrm{sea}}^{2}\right) .
$$

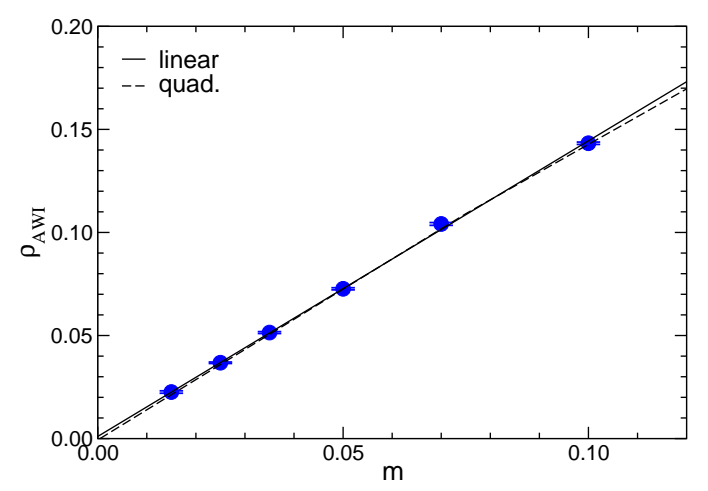

Figure 5: Chiral extrapolation of ratio $\rho_{\mathrm{AWI}}$ defined in Eq. (5.2).

The intercept $a_{\mathrm{AWI}}$ gives a measure of the chiral symmetry breaking. We confirm that results from linear $(0.0010(7))$ and quadratic fits $(-0.0007(9))$ are consistent with zero.

The slope $b_{\text {AWI }}$ provides a non-perturbative estimate of the renormalization constant $Z_{A}=$ $2 / b_{\text {AWI }}$. We obtain 1.394(20)(81), where the first error is statistical, and difference in $Z_{A}$ between the linear and quadratic extrapolations is added as a systematic error.

In Fig. 6, we plot our results for the pseudoscalar decay constant together with our previous estimates with the $O(a)$-improved Wilson quarks [1]. All results are renormalized nonperturbatively by $Z_{A}=2 / b_{\text {AWI }}$ or $Z_{A}$ in Ref.[19]. At heavy quark masses, we observe a reasonable consistency between our new and previous results with different discretizations. This suggests that these data are close to their continuum limit and have small scaling violations.

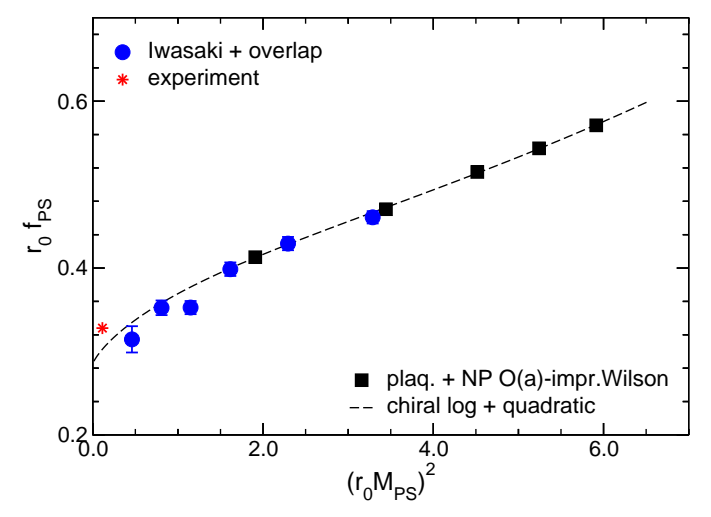

Figure 6: Chiral behavior of $f_{\mathrm{PS}}$. Squares are previous results with $O(a)$-improved Wilson quarks [1]. mass dependence is not smooth probably due to the limited statistics. As a result, chiral extrapolation with the chiral logarithmic term is very unstable. At this moment, it is difficult to make a definite conclusion on the consistency with chiral perturbation theory.

\section{Summary}

In this article, we report on the JLQCD's new project of large-scale simulations with dynamical overlap quarks. With careful choice of the lattice action and implementation of various algorithmic 
techniques, we demonstrated that it is feasible to accumulate high statistics on reasonably fine $(a \approx 0.125 \mathrm{fm})$ and large lattices $(L \approx 2 \mathrm{fm})$.

Our simulations have just started. We are currently accumulating $O(10,000)$ trajectories for a precise determination of meson masses and decay constants, with which we are going to investigate the consistency with chiral perturbation theory.

One of the most important subjects in the near future is the systematic effects due to the fixed topology. Simulations with $Q \neq 0$ are underway to check $Q$ dependence of physical observables. We are also planning to extend our simulations to three-flavor QCD. Pushing simulations toward larger volumes is also interesting future direction to explore the baryon sector as well as to check FSEs due to the small sea quark masses and the fixed topology.

Numerical simulations are performed on Hitachi SR11000 and IBM System Blue Gene Solution at High Energy Accelerator Research Organization (KEK) under a support of its Large Scale Simulation Program (No. 06-13). This work is supported in part by the Grant-in-Aid of the Ministry of Education (No. 13135204, 13135213, 13135216, 15540251, 16540228, 16740147, 16740156, 17340066, 17540259, 17740171, 18034011, 18104005, 18340075, 18740167).

\section{References}

[1] S. Aoki et al. (JLQCD Collaboration), Phys. Rev. D68 (2003) 054502.

[2] T. Ishikawa et al. (CP-PACS and JLQCD Collaborations), PoS (LAT2006) 181.

[3] N. Yamada et al. (JLQCD Collaboration), PoS (LAT2006) 060.

[4] Z. Fodor, S.D. Katz and K.K. Szabo, JHEP 0408 (2004) 003.

[5] P.M. Vranas, arXiv:hep-lat/0001006; hep-lat/0606014.

[6] H. Fukaya et al. (JLQCD Collaboration), arXiv:hep-lat/0607020.

[7] S. Hashimoto et al. (JLQCD Collaboration), PoS (LAT2006) 052.

[8] H. Fukaya et al. (JLQCD Collaboration), PoS (LAT2006) 050.

[9] A. Frommer et al., Int. J. Mod. Phys. C6 (1995) 627.

[10] N. Cundy et al., Comput. Phys. Commun. 165 (2005) 221.

[11] M. Hasenbusch, Phys. Lett. B519 (2001) 177.

[12] J.C. Sexton and D.H. Weingarten, Nucl. Phys. B380 (1992) 665.

[13] M.J. Peardon and J. Sexton, Nucl. Phys. (Proc.Suppl.) 119 (2003) 985; A. Ali Khan et al. (QCDSF Collaboration), Phys. Lett. B564 (2003) 235; C. Urbach, K. Jansen, A. Shindler and U. Wenger, Comput. Phys. Commun. 174 (2006) 87.

[14] H. Matsufuru et al., (JLQCD Collaboration), PoS (LAT2006) 031.

[15] R.G. Edwards et al., PoS (LAT2005) 146.

[16] R. Sommer, Nucl. Phys. B411 (1994) 839.

[17] F.C. Hansen and H. Leutwyler, Nucl. Phys. B350 (1991) 201.

[18] R. Brower, S. Chandrasekharan, J.W. Negele and U.-J. Wiese, Phys. Lett. B560 (2003) 64.

[19] M. Della Morte et al. (ALPHA Collaboration), JHEP 0507 (2005) 007. 